\title{
Acquired Feminization
}

National Cancer Institute

\section{Source}

National Cancer Institute. Acquired Feminization. NCI Thesaurus. Code C78299.

The development of secondary female sex characteristics in males due to extrinsic factors. 